Result $55 \%$ of the population were men; the median age was of 32 years. $51 \%$ were residents and $49 \%$ were doctors attached. There were significant differences in the working conditions of residents and doctors, $p<0.001$. There was higher prevalence of stress in residents than in doctors, $p=0.006$ The prevalence of emotional exhaustion was $44 \%$ for residents and $23 \%$ in doctors, $p<0.001$. The level of depersonalization was $51 \%$ and $46 \%$, respectively, $\mathrm{p}=0.004$, these had significant differences according to the working conditions. The risk of presenting severe psychosomatic manifestations was 3.5 times in those who had a high stress level (CI: 2.45 to 5.33 ). There were three times more risk for severity of symptoms in who perceived supervision (CI: 1.49 to 2.9) and excessive workload (CI: 2.06 to 4.11). The risk of burnout was twice for those who reported excessive workload and for those who performed guards and attend more than 20 patients per day. These differences were maintained by adjusting them by category on the job.

Discussion We found significant differences in the levels of stress, burnout and severity of symptoms according to the category and working conditions between residents and doctors attached.

\section{THE PSYCHOSOCIAL INTERVENTION AT THE WORKPLACE: PRACTICAL ADVANCES FOR SOLVING THE NEEDS OF COLOMBIAN WORKERS AND EMPLOYERS}

Gloria Villalobos. Universidad Javeriana. Bogotá Colombia

\subsection{6/oemed-2018-ICOHabstracts.1635}

Introduction The country's developments in occupational health, as well as the advances in the construction and use of instruments to evaluate the psychosocial factors (PF), opened the opportunity to advance in a project with the objective to develop a set of technical tools that will allow employers, workers, state and social security bodies to intervene psychosocial factors in work environments, so as to promote protective conditions and prevent the most important adverse effects impacting workers' health.

Methods An extensive review and analysis of literature was conducted to design the protocols to intervene the PF and their effects. Based on this information and considering the country's needs, the general protocols were designed and subsequently the specific protocols for intervention in different economic activities and their effects. Several sessions with experts and various stakeholders to discuss get feedback on the protocols were held.

Results In total, 13 protocols were designed:

- A general protocol with 34 intervention actions aimed at promoting protective PF and promotion of health.

- Specific protocols for psychosocial intervention by priority economic sectors (6).

- Specific intervention protocols (6) for the most important effects of psychosocial risk factors in the Colombian working population.

- General Technical Guide with complete procedures for the intervention.

Discussion Psychosocial intervention in the field of the workers' health is a necessity and its development involves combining actions focused both on the individual and the organisation. Colombia has been acknowledged in the Latin American region for issuing and adopting public policies related to providing care to psychosocial factors at the workplace, and also for developing technical tools for assessing psychosocial risk factors, and to determine the origin of stress-related diseases.

Intervention protocols help employers and workers progress significantly in improving working conditions, and they also support issuing public policies to enforce their mandatory application.

\section{ACTIVE JOBS ARE ASSOCIATED WITH BETTER COGNITIVE FUNCTIONING: FINDINGS FROM A POPULATION-BASED LONGITUDINAL STUDY IN GERMANY}

${ }^{1,2}{ }^{2}$ Natalia Wege, ${ }^{3}$ Hans Bosma, ${ }^{1}$ Peter Angerer, ${ }^{1}$ Jian Li. ${ }^{1}$ Institute of Occupational, Social and Environmental Medicine, Centre for Health and Society, Faculty of Medicine, University of Düsseldorf, Düsseldorf, Germany; ${ }^{2}$ Department of Psychiatry and Psychotherapy, Faculty of Medicine, University of Düsseldorf, Düsseldorf, Germany; ${ }^{3}$ Department of Social Medicine, Maastricht University, CAPHRI, Maastricht, the Netherlands

10.1136/oemed-2018-ICOHabstracts. 1636

Introduction Active jobs, which are the combination of high job demand and high job control based on Karasek's DemandControl model, have been found to be associated with better cognitive functioning by a couple of longitudinal studies. However, the evidence from Germany is still lacking. The purpose of our study is to analyse the associations of active jobs and cognitive functioning based on large German working population.

Methods We used data from the German Socio-Economic Panel (G-SOEP) Study with national representativeness. Two well-established cognitive tests (perceptual speed and word fluency) were applied in both 2006 and 2012. Psychosocial work conditions in terms of demand and control were measured in 2001. In total, longitudinal data from 1903 employees during follow-up 2001-2006, and from 1226 employees during follow-up 2001-2012 were available for current analyses. Multivariate linear regression was applied to examine longitudinal associations of psychosocial work conditions with cognitive functioning, adjusted for relevant covariates.

Results Overall, in this study sample (mean age 42.24 years, ranged 18-64 at baseline), participants with active jobs in 2001 exhibited the highest cognitive performance in 2006 and in 2012 compared to participants with passive jobs (low demand and low control), after adjustment for demographics, socioeconomic status, lifestyle behaviours, and health at baseline. Specifically, perceptual speed was higher 2.27 units $(95 \%$ confidence interval, CI: 0.54 to 4.00 ) in 2006, and 1.96 units (95\% CI: 0.77 to 3.16) in 2012; word fluency was higher 1.99 units $(95 \% \mathrm{CI}=0.27,3.72)$ in 2006 , and 3.55 units (95\% CI: 1.15 to 5.95 ) in 2012.

Conclusion This study demonstrates a protective effect of active jobs, defined as high demand and high control, on two aspects of cognitive functioning over an 11 year period. Further research is needed to determine the possible mechanisms and interventions, to maintain cognitive functions of employees. 